\title{
Cuatro poemas de amor y un elogio cortés: poesía catalana inédita de los siglos XV-XVI
}

\author{
Joan MAHIQUES CLIMENT \\ Institut National d'Histoire de l'Art \\ Groupe d'Anthropologie Historique de l'Occident Médiéval \\ jomahiques@yahoo.es
}

\begin{abstract}
RESUMEN
Partiendo de un estado de la cuestión sobre las diferentes aportaciones bibliográficas relativas a la poesía catalana medieval, se presenta el Repertori d'Obres en Vers (ROV) y se ofrece la edición de cinco poemas de los siglos XV-XVI hasta el momento inéditos, cuyos primeros versos son los siguientes: "Per sospirar trobe aleujament", "Dir-vos-ho he -sia secret-", "Ara·n lo punt de ma partida", "En tal cas astich posat" y "En totes parts on gentilesa's nota". Todos estos poemas son anónimos y se han conservado en un único testimonio manuscrito.
\end{abstract}

Palabras clave: Poesía catalana inédita, tradición extravagante, amor cortés, Edad Media.

[Recibido, febrero 2011; aprobado, junio 2011]

Four love poems and a courtois elogy: unpublished Catalan poetry from the $15^{\text {th }}$ and $16^{\text {th }}$ centuries

\begin{abstract}
Starting from a state of affairs of different bibliographical contributions on medieval Catalan poetry, this article presents the Repertori d'Obres en Vers (ROV) and includes the edition of five unpublished poems of the fifteenth and sixteenth centuries, whose opening lines are: "Per sospirar trobe aleujament", "Dir-vos-ho he -sia secret-", "Ara·n lo punt de ma partida", "En tal cas astich posat" y "En totes parts on gentilesa·s nota". All these poems are anonymous and have been preserved in a single manuscript.
\end{abstract}

Keywords: Unpublished Catalan poetry, "extravagant" tradition, courtly love, Middle Ages. 


\section{Poesía catalana medieval inédita: un estado de la cuestión}

El amplio corpus de la poesía catalana medieval, documentada sobre todo a lo largo de los siglos XIV-XV, cuenta con una consolidada tradición de estudios bibliográficos y filológicos que arranca como mínimo de principios del siglo $\mathrm{XX}$. En esta época, pero ya durante las dos últimas décadas del siglo anterior, Jaume Massó i Torrents publicó diversos catálogos de manuscritos, donde se daba a conocer el trabajo de campo realizado en algunas bibliotecas patrimoniales de ciudades como Barcelona, Madrid, Tarragona, Valencia o Vic. En el ámbito de la poesía catalana, la publicación de la "Bibliografia dels antics poetes catalans" (Massó i Torrents 1913-1914a) constituye un verdadero hito. Poco después, esta contribución inicial fue acompañada de una adición (Massó i Torrents 1913-1914b) y, dos décadas más tarde, fue sintetizada, revisada, ordenada y completada en el Repertori de l'antiga literatura catalana, del cual se publicó solamente el volumen dedicado a la poesía (Massó i Torrents 1932).

No hace falta insistir en el peso que han tenido las aportaciones de Massó en el ámbito de la historiografía literaria del siglo XX. ${ }^{1}$ Es suficiente recordar que, en una fecha mucho más reciente, el Repertori mètric de la poesia catalana medieval de Jordi Parramon i Blasco (1992) incorporaba también -a manera de índice para facilitar la búsqueda- un Repertori d'Autors i Obres (RAO) que todavía tomaba como punto de referencia el precedente del año 1932. En todo caso, el RAO tiene el interés de ordenar las obras escritas en verso, asignando a cada una de ellas o bien una edición de referencia o bien -en el caso de las obras inéditas- la fuente manuscrita. La consulta del RAO vendría a confirmar que, en términos generales, la mayor parte de la poesía catalana medieval ya había sido editada en el año 1992, cuando no antes, aunque esta constatación tampoco excluye el hecho de que entonces todavía había un buen número de poemas inéditos.

Sí que podemos decir que el inventario del RAO destaca por su amplitud, pues abarca un corpus de obras muy extenso, pero no podemos atribuirle el sello de la exhaustividad, porque desconoce poemas que entonces ya habían sido publicados. Por otra parte, como veremos a continuación, durante las últimas décadas se ha ido ampliando el conocimiento de nuestro patrimonio poético con el descubrimiento de nuevos testimonios manuscritos. A su vez, las inagotables posibilidades de la informática y de internet han facilitado el acceso a las fuentes y a los textos de la literatura medieval.

Partiendo del sistema de clasificación del RAO, el Repertorio Informatitzzato della antica letteratura catalana (Rialc 2011) ha dado a conocer en red no solamente muchas de las obras entonces conocidas sino también un buen número de textos inéditos hasta el momento, como se da de hecho en tres poesías ca-

\footnotetext{
${ }^{1}$ Para un análisis de los diferentes repertorios de poesía catalana medieval, remitimos a la introducción de nuestra tesis doctoral (Mahiques Climent 2009), hasta el momento inédita, de donde extraemos una buena parte de la información bibliográfica que citaremos en las páginas sucesivas de este artículo.
} 
talanas copiadas en el ms. 1 (olim 57) de la Lehigh University Library, en Bethlehem (Pennsylvania). ${ }^{2}$ En otros casos, el Rialc ha dado a conocer el texto íntegro de composiciones que hasta entonces se conocían solamente a través de ediciones parciales. ${ }^{3}$

Otra base de datos que a través de la web ha facilitado la localización de numerosos testimonios y poemas desconocidos es la Bibliografia dels textos antics catalans, valencians i balears (Biteca 2011). Aunque el objetivo de Bite$c a$ no es la edición de textos sino la localización y descripción de fuentes manuscritas o impresas, algunos de los investigadores vinculados a este proyecto han aprovechado el hallazgo de poemas totalmente desconocidos (o muy poco conocidos) para divulgarlos a través de diversas publicaciones. El intercambio de información entre el Rialc y Biteca también ha beneficiado a ambos proyectos, que se han ido enriqueciendo mutuamente con las aportaciones de cada uno.

Así mismo, el proyecto de Biteca ha sido respaldado con la colaboración de investigadores que, del mismo modo que han completado y revisado la información inicial de la base de datos, a su vez se han visto favorecidos, no solamente por las posibilidades que ofrece dicha base de datos para la selección y ordenación de los materiales sino también por el acceso a toda la información debida a los demás colaboradores del proyecto. En este aspecto, debemos seña-

\footnotetext{
${ }^{2}$ En los ff. 151-152 $2^{v}$ de este manuscrito se copian tres obras en verso ("En nom de Déu, lo rey del cel", "O! vosaltres, honrats senyors" y "Aujats, senyors, complits de saviesa") que aparecen en el Rialc, según una edición fechada en el año 2001 a cargo de Miriam Cabré y Michela Pereira. También pueden añadirse otros poemas que el Rialc ha dado a conocer en una primera edición surgida entre los años 2000-2001: "Com lo que sta sentensiat a mort" (copiado en el ms. 10 de la Biblioteca de Catalunya), "En nom de Déu totpoderós" (copiado en el volumen 235 de la notaría particular de Francesc Puig, en el Archivo Capitular de Barcelona), "Sanguini és joyós" (copiado en el ms. 18060 de la Biblioteca Nacional de Madrid), "Tant só devot contemplant vostra vida" (copiado en el ms. 210 de la Biblioteca de la Universidad de Valencia) y "Tothom qui sia ordonat" (copiado en el ms. 178 / fragmentos de códice 1 / núm. 16; en el Archivo Capitular de Barcelona).

3 Antes del año 2001, cuando el Rialc publicó "Qui fo ab ells" a cargo de Gemma Navarro, este poema (copiado en el ms. 759 de la Biblioteca de la Universidad de Barcelona) fue transcrito de manera fragmentaria por Jaume Massó i Torrents (1932: 489-490) i por Joan Ors (1983: 416). Otro caso que podemos mecionar es el de "Sapiats, sènyer En Ricard", cuyo único testimonio conocido nos ha llegado a través del ms. 490 de la Biblioteca de la Catalunya: Pere Bohigas i Balaguer (1920-1922: 44-45) se limita a transcribir tres fragmentos que en total suman 18 versos; mientras que, a través del Rialc, en el año 2001, Gemma Brunat da a conocer el poema íntegro -de 208 versos-.

${ }^{4}$ Es el caso de los tres poemas copiados en los ff. $14^{\mathrm{v}}-15^{\mathrm{v}}$ del ms. Palat. 1052 de la Biblioteca Nazionale Centrale de Florencia (Biteca 2011: manid 2673). Los tres poemas ("Na dolça res, bé m'és greu", "Bon esforç mal astre venç" y "Domna de bon ayre") han sido editados en el Rialc por una de las investigadoras que está al cargo de la dirección de Biteca, Gemma Avenoza, que ha publicado también un estudio y edición del primero de los tres (Avenoza 2006b). La pastorela comenzada "Ai espiga novela" también ha pasado al Rialc a través de Avenoza (2001). Otros tres poemas inéditos publicados por el grupo de Biteca, en un trabajo realizado por Grapí / La Marnierre (2001), han sido incorporados a la web del Rialc. Nos referimos a "La Verge molt singular" (copiado en el ms. 308 de la Biblioteca de Catalunya), "Sen Nicholau" (copiado en el ms. 93 de la Biblioteca de Catalunya) y "Tembre Déu é[s] saviesa" (copiado en el ms. 4030 de la Biblioteca Nacional de Madrid). A su vez, el corpus del Rialc ha sido revisado a fondo e incorporado a Biteca a partir de numerosas referencias cruzadas que en gran medida hemos realizado nosotros mismos y que quedan reflejadas en nuestra tesis doctoral (Mahiques Climent 2009).
} 
lar la redacción de dos visiones de conjunto de la poesía catalana medieval realizadas a partir del corpus de Biteca.

Partiendo de la clasificación establecida por Massó i Torrents (1932) y completándola, Gemma Avenoza (2006a: 112-124) ofrece una tabla de los manuscritos que han transmitido la poesía catalana medieval. Esta tabla, que parte de la información de Biteca y refleja el estado de la cuestión de dicho corpus en el año 2006 o poco antes, es de gran utilidad, pues constituye el listado más exhaustivo que hasta ahora se ha publicado de las fuentes manuscritas de la poesía catalana medieval.

También parte de Biteca el trabajo que nosotros hemos presentado como tesis doctoral (Mahiques Climent 2009), donde, además de ampliar y corregir el listado de Avenoza (2006a), se incorpora el Repertori d'Obres en Vers (ROV), un catálogo de toda la poesía catalana medieval conocida hasta el momento, con el desglose detallado de todos los testimonios antiguos (impresos o manuscritos) de cada uno de los poemas en cuestión. El ROV también tiene la ventaja de consignar, según el caso que se de, o bien las ediciones de cada poema o bien las composiciones que son inéditas. De este modo, a partir del análisis de la información del ROV, hemos localizado un notable grupo de poemas inéditos hasta el momento. Este trabajo refleja el estado de la cuestión de Biteca al inicio del año 2009. ${ }^{5}$

\section{Edición de cuatro poemas de amor y un elogio cortés}

A partir de los datos que suministra el ROV, hemos seleccionado cinco poemas inéditos donde se recrean diversos tópicos de la lírica amorosa y cortés, como son el rechazo de la pasión amorosa o el elogio de la dama. Los poemas en cuestión son los siguientes:

(I) "Per sospirar trobe aleujament"

(II) "Dir-vos-ho he-sia secret-"

(III) "Ara·n lo punt de ma partida"

(IV) "En tal cas astich posat"

(V) "En totes parts on gentilesa·s nota"

Respetando este mismo orden, editaremos estos cinco poemas, conservados todos ellos en testimonios manuscritos únicos que desde el punto de vista cronológico oscilan entre la segunda mitad del siglo XV y la primera mitad de la

\footnotetext{
${ }^{5}$ El listado de manuscritos que acompaña al ROV parte de la tabla realizada por Avenoza (2006a), aunque diverge en lo referente al sistema de clasificación. Las aportaciones más novedosas que el ROV incorpora en relación a la crítica filológica precedente pueden rastrearse a partir de la consulta de dos tablas de nuestra tesis doctoral (Mahiques Climent 2009). Por una parte, bajo el epígrafe "Manuscrits del llistat d'Avenoza 2006a exclosos en el ROV", se introducen algunas enmiendas de ítems duplicados o de fuentes que en realidad no incluyen ningún poema, mientras que bajo el epígrafe "Manuscrits del ROV no inclosos al llistat d'Avenoza 2006a" se amplía considerablemente el corpus de manuscritos de la poesía catalana medieval.
} 
centuria siguiente. El último de los cinco poemas se distingue claramente del resto, pues se inscribe en la tradición del elogio cortés dirigido a una o a diversas damas. En este género de poemas, las damas ensalzadas suelen ser de elevada posición social, y no deben confundirse con la amada a quien suele dirigirse la poesía amorosa. El elogio cortés incide sobre todo en la virtud de la gentilesa, sin olvidar el nombre propio de la dama o las damas en cuestión, como de hecho sucede en un acróstico de "En totes parts on gentilesa·s nota", donde podemos leer "Evlalya". 6

A estos cinco poemas, cuyos originales manuscritos hemos consultado personalmente, debería de añadirse una esparsa que se ha copiado a línea seguida en el f. $33^{v}$ del ms. 97-23 de la Biblioteca del Cabildo de Toledo. Se trata de una fuente que, a parte de ser descrita en Biteca (2011: manid 1940) y de incorporarse al ROV, ha merecido la atención de Font i Sagué (1908: 60-61) y de Millás Vallicrosa (1942: 121-125, ítem xxviii). La lectura realizada a partir de una reproducción nos ha permitido observar que el incipit del poema que hasta ahora han ofrecido tanto Biteca como el ROV ("cas pareys eu pris lamoros amans") es el resultado de una lectura errónea, que debe corregirse por la siguiente: "Eras pareis eu pris lamoros ams". ${ }^{7}$ La escasa calidad de la reproducción de que disponemos, junto con el hecho de que hasta el momento no hayamos consultado personalmente el original, nos ha hecho desistir en el intento de editar esta esparsa.

Continuemos con los cinco poemas que sí que editaremos en las páginas siguientes. A la edición de cada uno de ellos precede la localización y una des-

\footnotetext{
${ }^{6}$ En el ámbito de la poesía catalana del siglo XV, Andreu Febrer y Joan Berenguer de Masdovelles son dos de los autores que más claramente se inscriben en la tradición del elogio dirigido a mujeres de la alta nobleza. Véanse los comentarios de Martí de Riquer a "Si·n lo món fos gentilesa perduda" de Andreu Febrer (1951: 8486 y 136-138), y a "A vós, qui sou de complida ballesa" de Joan Berenguer de Masdovelles (Riquer 1993: III, 520-521). La tradición del elogio cortés con acrósticos que desvelan el nombre de la dama pervive todavía en poetas del siglo XVI como Pere Serafí (2001: 290-319) o Joan Pujol. Este último es autor de una composición en castellano todavía inédita que comienza "Ferós, sin consuelo y sañuda dama", copiada en el f. $139^{\mathrm{r}-\mathrm{v}}$ del ms. 4495 de la Bibliothèque Mazarine de París. En la primera octava de este poema se lee el acróstico "Francyna", mientras que en cada verso (justo en el lugar de cesura) se lee, según el recurso del calambur, otro nombre femenino. Por ejemplo, leemos en el primer verso "consueLO Y SAñuda", y en el segundo "trabaJO A NAdie". De este modo podemos observar que los recursos retóricos del último de los cinco poemas que editaremos entran de lleno en el género del elogio cortés.

${ }^{7}$ Deberíamos añadir este poema a la tradición de las obras que selecciona la misma palabra de inicio, como sucede en las siguientes composiciones anónimas: "Eras diray ço que us dey dir", "Eras mi ponch Amors tan finamen", "Eras quant vey arbres e brotz florir", "Eres quan vey los arbres gen florir". O como también se da de hecho en otras poesías de autores como Joan Berenguer de Masdovelles ("Eras can vey guarnits los pratz e-ls camps", "Eras, pus ssuy ssí fort anamorats", "Eres maldich lo jorn, lo punt e l'ora", "Eres plus vey c'Amors vol que sieus sia"), Lluís Icart ("Eras quant vey dels brots tombar la flor") y Gilabert de Pròixita ("Eras quant torn lay on és mon voler"). Debemos añadir, además, que en el último verso de la esparsa conservada en el manuscrito toledano se reitera esta misma expresión: "Eras am trist doloros e ploran". Cosa que sucede también en un poema anónimo del Cançoneret de Ripoll, iniciado "Ayman suy Bel Joy e l'alba" y acabado "Eras dyats si n'e colpa" (Badia 1983: 183-187). Estos poemas se han transmitido en su mayor parte a través de cancioneros cuyo marco cronológico va del siglo XIV a la primera mitad del siglo XV, como sucede con el Cançoneret de Ripoll (ms. Ripoll 129 del Arxiu de la Corona d'Aragó) o el Cançoner Vega-Aguiló (ms. 7 de la Biblioteca de Catalunya). Para una descripción más detallada de cada uno de los poemas y manuscritos hasta ahora citados, remitimos a Mahiques Climent (2009).
} 
cripción muy breve del manuscrito que lo transmite y de la bibliografía crítica pertinente: toda esta información la hemos extraído de Mahiques Climent (2009). También indicamos el esquema métrico de la composición y, siempre que es necesario, un aparato crítico donde queda consignada cualquier divergencia existente entre el testimonio base y la edición. Por tanto, la consulta del aparato crítico es indispensable para la localización de las correcciones que la edición aplica al testimonio manuscrito. Por lo que se refiere a las normas de transcripción de los textos, hemos mantenido los criterios más usuales entre editores de textos catalanes medievales. Resolvemos las abreviaturas y regularizamos el texto -según los usos modernos- en las alternancias entre $i / j$ y $u / v$, en la separación de las palabras y en el uso de mayúsculas y minúsculas. Seguimos las normas ortográficas catalanas actuales de acentuación, diéresis, guiones y apóstrofes. Marcamos con un punto volado las elisiones que hoy no tienen representación gráfica, y entre corchetes la parte del texto editado que falta en el testimonio manuscrito.

Después de esta breve introducción, continuaremos con la edición de los cinco poemas que acabamos de presentar. 
MANUSCRITO: Trinity College Library (Cambridge), ms. R.14.17 (olim B.7.3). El poema ocupa el f. $123^{\mathrm{v}}$ de este manuscrito, datado del inicio del siglo XVI, y estudiado en Annichiarico (2004: 444-445, ítem A), Biteca (2011: manid 1324), Bohigas (1985: 37-42), Ganges (1992: 75-76, ítem 10), March (1997: II, 19, ítem $\mathrm{O}=\mathrm{X}^{2}$ ), Martos (1999), Martos (2005), Massó i Torrents (1932: 23-24, ítem X²), Roós de Corella (1984-1985: I, 15-17) y Serra i Baldó (1936: 380-384).

ESQUEMA MÉTRICO: 4 × 8 (1: ABBACCDD; 2-4: EFFEGHHG...). Tendencia al uso del decasílabo catalán con cesura masculina (y, en algún caso, femenina) en la cuarta sílaba, aunque se localizan versos hipométricos e hipermétricos. Rimas masculinas y femeninas. Aunque en la tradición poética catalana es más usual la combinación de rimas de las estrofas 2-4 que las de la cobla inicial, se pueden aducir numerosos casos del esquema métrico de esta última. Véanse los diferentes testimonios de ambas combinaciones en Parramon i Blasco (1992: $137-142$ y $152-200$, ítems 200 y 219 ).

APARATO CRÍTICO: 28 perdent] sabent perdent.

\section{PLANY}

Per sospirar trobe aleujament

a mes dolors e penes insesables.

Totes les dones veig ésser variables;

no senten pena ne senten greu turment.

E tinch ja per foll qui en amor se met,

car despèn lo temps e no $n$ trau lo bestret

e torna foll si molt hi vol pensar.

A tothom prech que no vulga amar.

Tot bon treball deu ésser ben pagat

segons rahó e juhí natural.

Tostemps me veig anar de mal en mal;

del que yo fas no $\mathrm{m}$ veig remunerat.

Pas gran treball e pert lo meu jovent;

no cur de res sinó de contemplar

e'n foch amorós me veig forment cremar:

passa lo temps, e va-se'n com a vent.

Renech d'amor, puix no'm vol consentir

que 1 desamar sia.n ma libertat, ans com més va més me trobe ligat 
de tals ligams que no.ls puch resistir, cremant d'un foch que no ha par; que, puix és ensès, no y ha remey que l'apagàs aprés per molt amor, que no's pot oblidar.

Consell vos do a tots en general: ab lealtat no ameu si voleu viure, car tant vos val com en l'aygua scriure, perdent lo temps e, puix, vostre treball. Dich-ho per tant com no han fermetat. Totes són tals, sols han parenceria. Alégran-se com vos han dit falçia. Vostre treball los és stat oblidat. 
MANUSCRITO: Biblioteca Nacional de Madrid, ms. 3066 (olim L.12). Este manuscrito es descrito en Biteca (2011: manid 1246), Domínguez Bordona (1931: 41), Dutton (1990-1991: II, 22), Hitos Natera (1968: 61-62) e Inventario (1953-2001: X, 11). Por el tipo de letra, consideramos que la copia de la carta y el poema que a continuación editamos, localizada en el f. $108^{\mathrm{v}}$ del manuscrito, data de la segunda mitad del siglo XV. Como en el testimonio manuscrito no figura ninguna rúbrica inicial, hemos incorporado en nuestra edición un título entre corchetes, acorde con el contenido de la obra.

ESQUEMA MÉTRICO: $1 \times 5,1 \times 9,1 \times 5,2 \times 9\left(1+3\right.$ : $\mathrm{A}^{8} \mathrm{~B}^{8} \mathrm{~B}^{4} \mathrm{~B}^{4} \mathrm{~A}^{8} ; 2+4-5$ : $\left.\mathrm{C}^{8} \mathrm{D}^{8} \mathrm{C}^{8} \mathrm{D}^{8} \mathrm{~A}^{8} \mathrm{~B}^{8} \mathrm{~B}^{4} \mathrm{~B}^{4} \mathrm{~A}^{8}\right)$. Todo el poema está compuesto en rimas masculinas, y con cesura masculina en la cuarta sílaba de todos los octosílabos. El v. 8 es hipométrico. Tras la consulta del Repertori mètric de Parramon i Blasco (1992), no hemos localizado ninguna obra que reproduzca el esquema métrico del poema que ahora editamos.

APARATO CRÍTICO DEL PASAJE EN PROSA: 1 dega] degi [?] || $\mathbf{2}$ senyora] senjora $\| \mathbf{3}$ dolça] dolca [?] || $\mathbf{4}$ cascuna] cascuda $\| \mathbf{5}$ per sso eng[...] són] per sso engosal [?] son; lectura conjetural \|| $\mathbf{6}$ pasaje ilegible; el texto ha quedado manchado por un exceso de tinta $\| \mathbf{7}$ bastans] batans [?] \| $\mathbf{8}$ sobrepuja] sobre puga $\| \mathbf{9}$ lectura conjetural $\| \mathbf{1 0}$ subrepuja] subre puga \|| $\mathbf{1 1}$ senyora] senjora $\|$ 12 seny] sey || 13 lectura conjetural $\| \mathbf{1 4}$ dolça] dolca \|15 lectura conjetural; la abreviatura del manuscrito (psent) debería resolverse como "present" más que como "pensament" || 16 voluntat] uluntat || 17 forçat] forcat || 18 lectura conjetural $\| 19$ senyora] senjora $\mid \mathbf{2 0}$ senyoria] senjorja $\| \mathbf{2 1}$ senyal] senjal || $\mathbf{2 2}$ senyoria] senjoria || $\mathbf{2 3}$ senyora] senjo || $\mathbf{2 4}$ senyoria] senjoria || $\mathbf{2 5}$ dolça] dolca || $\mathbf{2 6}$ senyoria] senjoria || 27 ço] co || $\mathbf{2 8}$ desige] dexige.

APARATO CRÍTICO DEL PASAJE EN VERSO: $\| \mathbf{2}$ vull] vult $\| \mathbf{3}$ penar] perar $\| 6$ Senyora, almenys] senjora almeys $\| \mathbf{7}$ que ab [...] a vos serveys] que ab contra la vo ler [?] auos seruens; lectura conjetural $\| \mathbf{8}$ pes] lectura conjetural, de dificil interpretación; el manuscrito transcribe: pes; o bé: pos \| 9 qu·eu] quer [?] || 20 sapiau] lectura conjetural; el manuscrito transcribe: sa plau; $o$ bien: sa piau || 22 pijor] pi gor || $\mathbf{2 3}$ Desde el punto de vista morfológico, consideramos esta palabra como una forma personal del verbo catalán "haver" || $\mathbf{2 5}$ jorns] lectura conjetural: jorns jors jorns [?] || 27 e vul] enul [?] || 28 trist] trest || 28 / 29 endreça] entre || 29 Que] ques || 30 lo qual] la qual || $\mathbf{3 4}$ jo] jo non $\| \mathbf{3 5}$ deus] deus et [?].

[REQUESTA D'AMOR] 
Com per cascun he ha força de gentilesa tota persona requesta axí de armes com de amós ab tota eficàcia dega ${ }^{1}$ aceptar tal requesta com vós, senyora ${ }^{2}$ magnada, siasts aquela la qual, per les grans excel·lències, virtuts he gentileses qui de vostra he insigne persona dolça $^{3}$ incesanment eixent hi procehixent. Les quals dir e explicar cascuna ${ }^{4}$ de aqueles per sso eng[... $]^{5}$ són forzat calar; com mon seny, saber, sentiment ne enginy no sien bastans ne suficiens a compenre ne jamés manifestar ni explicar aqueles; com tota persona $[. . .]^{b}$ bas$\operatorname{tans}^{7}$ perfeció natural Déus haye volgut fabricar e demostrar en vostra dolça, insigne persona dotada de natura, favorida per fortuna he amada per gentilesa.

Car axí com lo sol per la sua grant excel-lència sobrepuja ${ }^{8}$ hi apag[u]e totes les altres clarós, axí pròpriament vostra ssí ${ }^{9}$ dolça persona subrepuja ${ }^{10}$ hi hapag[u]e les excel-lèncias, virtuts hi gentileses de totes les altres persones; per les quals coses, senyora ${ }^{11}$ magnada, mon cor, mon cos, mon seny ${ }^{12}$ hi saber e tots mons sentimens són axí constrests e comanasts ${ }^{13}$ en amar, obeir, duptar e cartenir vostra dolça ${ }^{14}$ hi insigne persona; que per nenguna via hi manera per mi no pot ésser feta resistència ne contradicció alguna en mudar pensament $^{15}$ ne voluntat ${ }^{16}$, ans són axí forment astret e forçat ${ }^{17}$ que·n puxque $^{18}$ del tot penant morir.

A vós, senyora ${ }^{19}$ magnada, de fina amor, de cordial amor fas la present humilment e devota requerir a vostra dolça senyoria, que per la vostra acustumada gentilesa vullasts ab tota eficàcia ma humil hi devota requesta admetre aceptar axí com, per deute, stil de gentilesa devest fer, suplicant axí humilment com dir ne fer se pus[c]a a vostra dolza senyoria ${ }^{20}$ que, de indigne que só, me vulats fer digne vassal e sosmès vostre, en senyal ${ }^{21}$ de fealtat que tot vasal obtengut a sa senyoria ${ }^{22}$; ara de present renun[ci]ans, senyora ${ }^{23}$ magnada, ha tot altre for e juridicció; sosmetent ma persona a tota voluntat en nom e veu de vostra dolza senyoria ${ }^{24}$. La qual humilment requerir per deure de gentilesa que de les coses per mi damunt requestes haye vostra dolça $^{25}$ hi graciosa resposta, confiant per aquela obtenir la fi de mont grant desig. E man ha mi vostra dolza senyoria ${ }^{26}$ tot ço $^{27}$ he quant plasent la sie.

Scrit de la mia mà ha no sé quant de present mes, lo qui ab grant desig desige ${ }^{28}$ a ser vasal hi sobmès vostre.

Dir-vos-ho he -sia secret-; amor, mos mals vos vull contar, qui.m fan penar

e confesar:

ay trist de mi, he què $[\mathrm{u}] \mathrm{s}$ he fet? 
Co[bla]

Senyora, almenys, com no pensau

que ab $[\ldots]$ a vos serveys

e com hun pes pasegau

per los meus mals en qu'eu pereys?

Lo primer és cas ben stret;

que $a b$ vós no pusc jamés parlar

ne demostrar

mon voler clar.

Ay trist de mi, he què $[u] s$ he fet?

\section{Tornada}

Cor virtuós a vós tramet.

Aquests tres mals v[u]lau mirar

que'm feu pasar

sens remeyar.

Ay trist de mi, he què $[\mathrm{u}] \mathrm{s}$ he fet? $\left.\right|^{\mathrm{b}}$

Encara eu plant que u sapiau!

De plor lo dol may no.m portests.

De mal en mal en pijor vau.

Mon cor, qui és trist, he no gens fret.

Lo segon mal bé'n té constret

-las!- com tots jorns no us pug mirar.

Cuit esclatar

e vul plorar...

Ay trist de mi, he què $[\mathrm{u}] \mathrm{s}$ he fet?

\section{Endreça}

Que fort dolor cert me donau, lo qual jo -trist!-may jo jaqués, ans me té pres com hun sclau; ara no sé com me regés.

Lo tercer mal fort jo us promet.

$\mathrm{Si} \cdot \mathrm{n}$ voleu bé, com jo sé clar, bé deus cridar o sospirar.

Ay trist de mi, he què [u]s he fet? 
(III)

MANUSCRITO: Biblioteca de la Universidad de Barcelona, ms. 101 (olim 1-431). Hay una descripción de este manuscrito en Biteca (2011: manid 1074), Massot i Muntaner (1962-1967: 38-39, ítem 3) y Miquel Rosell (1958-1969: I, 123). El poema que ahora editaremos se ha conservado en un testimonio ápodo -en el f. $15^{\mathrm{v}}$ de este manuscrito- que consideramos copiado durante el último tercio del siglo XV. Como en el testimonio manuscrito no figura ninguna rúbrica inicial, en nuestra edición incorporamos entre corchetes el primer verso de la composición, a manera de título.

MÉTRICA: $1 \times 3,1 \times 6\left(1+2: \mathrm{A}^{8} \mathrm{~B}^{8} \mathrm{~A}^{8}+\mathrm{B}^{8} \mathrm{C}^{8} \mathrm{~B}^{8} \mathrm{C}^{8} \mathrm{~A}^{8} \ldots\right)$. Rimas masculinas y femeninas. El carácter fragmentario del poema no nos permite hacer una reconstrucción completa de su esquema métrico. De todos modos, puede constatarse sin demasiadas dificultades que se ciñe al patrón de la dansa. En particular, debe señalarse la semejanza formal con algunas de las composiciones que transmite el Cançoneret de Ripoll (ms. Ripoll 129 del Arxiu de la Corona d'Aragó), tal como puede observarse en el corpus reunido por Avenoza (2003: 102-103).

APARATO CRÍTICO: 4 forçat] forcat $\| \mathbf{7}$ en] lectura conjetural.

["ARA·N LO PUNT DE MA PARTIDA"]

Ara $n$ lo punt de ma partida, me són ten fort anamorat que, si me'n vaig, perdré ma vida.

Equest partir m'és ten forçat que aturar ya no poria.

$O$ déus Amor, bé m'as portat en gran peril la vida mia [...] puis partir e tot jorn crida $[\ldots]$ voler $[\ldots]$ 
MANUSCRITO: Biblioteca de la Societat Arqueològica Lul-liana (Palma de Mallorca), ms. A(M) 88. Este manuscrito es conocido como el Llibre de recorts del senyor Francesch Serralta, de 1521, y es descrito en Biteca (2011: manid 2375) y en Cirera / Le-Senne (1982: 158). El poema que editaremos a continuación ha sido copiado en la cubierta y, más concretamente, en el reverso del plano anterior de dicho manuscrito. Creemos que la escritura de esta composición no debe ser muy lejana a la del cuerpo del volumen, que es de 1521. Nuestra edición toma como base una transcripción paleográfica que nosotros mismos hicimos durante una consulta del manuscrito, ya hace algunos años. Al acabar la consulta quedaron pendientes algunas dudas que no han sido verificadas, pues no poseemos una reproducción del testimonio. Debemos advertir que las lagunas de nuestra edición señaladas entre corchetes no responden al estado del testimonio manuscrito, sino que indican los fragmentos de nuestra transcripción paleográfica que han quedado sin resolver. Como en el testimonio manuscrito no figura ninguna rúbrica inicial, en nuestra edición incorporamos entre corchetes el primer verso de la composición, a manera de título.

MÉTRICA: $1 \times 3,2 \times 7\left(1: X^{7} B^{7} B^{7} ; 2-3: C^{7} D^{7} C^{7} D^{7} D^{7} A^{7} A^{7} \ldots\right)$. Rimas masculinas en todos los versos. Aunque el esquema métrico podría asimilarse al de la dansa medieval estudiada por Avenoza (2003), creemos que este poema debe vincularse más directamente con el villancico o vilancet característico del siglo XVI. Remitimos, por ejemplo, a los poemas "O dichosa desventura" y "Puix si us mir vós me mirau" de Pere Serafí (2001: 191-192 y 196-198).

\section{["EN TAL CAS ASTICH POSAT"]}

En tal cas astich posat

que jo mateix no puch dir si só mort o a morir.

\section{Cobla}

Ocupats mos sentiments, [...]a tanta pasió, estan bevent los turments ab rasó o sens rasó. [...] gran compasió que de mi no puxa dir si só mort o a morir. 
No·[m] vullau matar axí, ajau de mi pietat.

Del prinsipi a la fi,

Sempre fael són [...]at.

O lo meu tresor amat, quant al món [...]xa dir, $a b$ vós vull viure y morir. 
(V)

MANUSCRITO: Biblioteca de Catalunya (Barcelona), ms. 1218. El poema ha sido copiado en el f. $40^{\vee}$ de este manuscrito, que data del final del siglo $\mathrm{XV}$, pueden consultarse las descripciones de Biteca (2011: manid 2419), Duran (1998: 265-266) y Guía (1959: 99). Estamos ante una esparsa dirigida a una dama llamada Eulalia, según se deduce de la lectura de los acrósticos.

MÉTRICA: $1 \times 8\left(\mathrm{~A}^{10} \mathrm{~B}^{10} \mathrm{~B}^{10} \mathrm{~A}^{9} \mathrm{C}^{10} \mathrm{D}^{10} \mathrm{D}^{10} \mathrm{C}^{10}\right)$. Cesura masculina en la cuarta sílaba, y rimas femeninas en todos los versos. Véase un listado de composiciones con este esquema métrico en Parramon i Blasco (1992: 152-200, ítem 219).

\section{UN DELIT GRAN QUE DI[U]}

En totes parts hon gentilesa's nota, Veyg divulgar vostre renom e ffama:

Lum dels sabents, qui tot vissi desama;

Arxiu d'onor, virginal e nota

Leal e prous, sabent e valerosa;

Yoia d'onor; miral de tota-btesa.

A vós me do, per vostra gentilesa, que dama sou de virtuts abundosa. 


\section{Referencias bibliográficas}

ANNICHIARICO, Annamaria (2004): "L'edizione critica delle Faules mitologiche di Joan Roís de Corella: bilanci, sondaggi, proposte", La parola del testo. Semestrale di filologia e letteratura europea dalle origini al Rinascimento 7/2: 443-466.

AVENOZA, Gemma (2001): "Ai espiga novela! Estudi i edició d'una pastorel·la inèdita catalana del s. XIV", en Studia in honorem Germán Orduna, Leonardo Funes y José Luis Moure (eds.), pp. 53-71. Alcalá de Heranes: Universidad de Alcalá.

AVENOZA, Gemma (2003): "La dansa. Corpus d'un genre lyrique roman", Revue des Langues Romanes 107: 89-129.

AVENOZA, Gemma (2006a): "Cancioneros catalanes: de los epígonos trovadorescos hasta el final de la Edad Media", en Convivio. Estudios sobre la poesía de cancionero, Vicenç Beltran y Juan Paredes (eds.), pp. 97-144. Granada: Universidad de Granada.

AVENOZA, Gemma (2006b): "Poemes catalanoocitans del s. XIV en un manuscrit florentí. Edició i estudi de $\mathrm{Na}$ dolça..., primer del recull", en Trobadors a la Península Ibèrica, Vicenç Beltran, Meritxell Simó y Helena Roig (eds.), pp. 73-90. Barcelona: Publicacions de l'Abadia de Montserrat.

BADIA, Lola (1983): Poesia catalana del s. XIV. Edició $i$ estudi del Cançoneret de Ripoll. Barcelona: Quaderns Crema.

BITECA (2011). BELTRAN, Vicenç / AVENOZA, Gemma / SORIANO, Lourdes (dirs.): Bibliografia de textos antics catalans, valencians $i$ balears, en Philobiblon. Berkeley: Bancroft Library. $<$ http://bancroft.berkeley.edu/philobiblon $>$

BOHIGAS I BALAGUER, Pere (1920-1922): "Profecies catalanes dels segles XIV i XV. Assaig bibliogràfic", Butlletí de la Biblioteca de Catalunya 6: 2449.

BOHIGAS, Pere (1985): Sobre manuscrits $i$ biblioteques. Barcelona: Publicacions de l'Abadia de Montserrat.

CIRERA, Jaime / LE-SENNE, Aina (1982): "Los libros manuscritos del 'Fondo Estanislao de K. Aguiló' de la Sociedad Arqueológica Luliana", Bolletí de la Societat Arqueologica Luliana 39: 145-164.

DOMÍNGUEZ BORDONA, Jesús (1931): Catálogo de los manuscritos catalanes de la Biblioteca Nacional. Madrid: Blass S. A. Tipográfica.

DURAN, Eulàlia (dir.) (1998): Repertori de manuscrits catalans (1474-1620). Vol. 1. Barcelona: Arxiu Històric i Biblioteca de Catalunya. Barcelona: Institut d'Estudis Catalans.

DUTTON, Brian (1990-1991): El cancionero del siglo XV, c. 1360-1520. Cancioneros musicales al cuidado de Jineen Krogstad, 7 vol. Salamanca: Universidad de Salamanca. 
FEBRER, Andreu (1951): Poesies. A cura de Martí de Riquer. Barcelona: Barcino.

FONT I SAGUE, Norbert (1908): Historia de les ciències naturals a Catalunya del sigle ix al sigle xviii. Premiada en el Concurs celebrat l'any 1905 per la Societat Económica d'Amichs del País de Barcelona. Barcelona: La Hormiga de Oro.

GANGES GARRIGA, Montserrat (1992): "Poetes bilingües (català - castellà) del segle XV", Boletín bibliográfico de la Asociación Hispánica de literatura medieval 6/1: 57-231.

GRAPÍ, Orland / LA MARNIERRE, Edith de (2001): "Al marge dels cançoners (1): Alguns textos poètics catalans inèdits o poc coneguts", en Canzonieri iberici, Botta, Patrizia y Carmen Parrilla (eds.), vol. I, pp. 219-265. Noia: Università di Padova / Toxosoutos / Universidade da Coruña.

GUÍA (1959): Guía de la Biblioteca Central de la Diputación Provincial de Barcelona. Barcelona: Casa Provincial de la Caridad.

HITOS NATERA, María del Pilar (1968): "Índice de los manuscritos existentes en la Biblioteca Nacional de Madrid de interés a la historia de la Farmacia y Ciencias Afines, con breves comentarios de su contenido" [primera parte], Boletín de la Sociedad Española de Historia de la Farmacia 19: 49-72.

INVENTARIO (1953-2001): Inventario general de manuscritos de la Biblioteca Nacional, 15 vol. Madrid: Dirección General de Archivos y Bibliotecas [vol. 1-9] / Dirección General del Libro y Bibliotecas [vol. 10-13] / Ministerio de Educación, Cultura y Deporte [vol. 14-15].

MAHIQUES CLIMENT, Joan (2009): Repertori d'Obres en Vers. Una aportació a la 'Bibliografia de Textos Catalans Antics'. Tesi Doctoral sota la direcció del Dr. Vicenç Beltran Pepió i de la Dra. Lola Badia Pàmies. Barcelona: Universitat de Barcelona / Departament de Filologia Catalana. [Tesis doctoral inédita, defensada el día 19 de mayo del año 2009].

MARCH, Ausiàs (1997). Obra completa, Robert Archer (ed.), 2 vol. Barcelona: Barcanova.

MARTOS, Josep Lluís (1999): "El còdex de Cambridge del Trinity College, R.14.17 ( $\left.\mathrm{X}^{2}\right)$ : descripció i estudi", en Actes del VII Congrés de l'Associació Hispànica de Literatura Medieval (Castelló de la Plana, 22-26 de setembre de 1997), Santiago Fortuño Llorens y Tomàs Martínez Romero (eds.), vol. II, pp. 443-460. Castelló de la Plana: Universitat Jaume I.

MARTOS, Josep Lluís (2005): "El Còdex de Cambridge, el Cançoner de Maians y el Jardinet d'orats a través de la obra de Roís de Corella", en Los cancioneros españoles: materiales y métodos, Moreno, Manuel y Dorothy Severin (eds.), pp. 113-140. London: Papers of the Medieval Hispanic Research Seminar.

MASSÓ I TORRENTS, Jaume (1913-1914): Jaume MASSÓ I TORRENTS, "Bibliografia dels antics poetes catalans", Anuari de l'Institut d'Estudis Catalans 5/1: 3-276. 
MASSÓ I TORRENTS, Jaume (1913-1914b): "Adició a la bibliografia dels antics poetes catalans", Anuari de l'Institut d'Estudis Catalans 5/2: 738-740.

MASSÓ I TORRENTS, Jaume (1932): Repertori de l'antiga literatura catalana. La Poesia: vol. I. Barcelona: Editorial Alpha.

MASSOT I MUNTANER, Josep (1962-1967): "Notes sobre la supervivència del teatre català antic", Estudis Romànics 11: 49-101.

MILLÁS VALLICROSA, Jose María (1942): Las traducciones orientales en los manuscritos de la catedral de Toledo. Madrid: Consejo Superior de Investigaciones Científicas / Instituto Arias Montano.

MIQUEL ROSELL, Francisco (1958-1969), Inventario general de manuscritos de la Biblioteca Universitaria de Barcelona, 4 vol. Madrid: Direcciones Generales de Enseñanza Universitaria y de Archivos y Bibliotecas.

ORS, Joan (1983): "Sobre l'extravagància del Poemet extravagant", Estudis Universitaris Catalans 25: 413-424.

PARRAMON I BLASCO, Jordi (1992): Repertori mètric de la poesia catalana medieval. Barcelona: Curial / Publicacions de l'Abadia de Montserrat.

RIALC (2011). DI GIROLAMO, Costanzo (coord.): Repertorio informatitzzato della antica letteratura catalana. <http://www.rialc.unina.it>

RIQUER, Martí de (1993): Història de la literatura catalana. Part antiga, 4 vol. Barcelona: Ariel.

ROÍS DE CORELLA, Joan (1984-1985): Obres de Joan Roiç de Corella, Josep Almiñana Vallés (ed.), 2 vol. Valencia: Del Cenia al Segura.

SERAFÍ, Pere (2001): Poesies catalanes. Edició crítica de Josep Romeu i Figueras. Barcelona: Barcino.

SERRA I BALDÓ, Alfons (1936): "Els 'Goigs de la Verge Maria' en l'antiga poesia catalana", Estudis Universitaris Catalans 22: 367-386. 